\title{
Rabid about whole lyssa genomes
}

\author{
Eva Archer and Charlotte J. Houldcroft
}

Sequencing of whole rabies virus genomes is revealing novel genetic variants that influence virulence and transmission.

Rabies virus (RABV) belongs to the lyssavirus genus, is spread by saliva and for both animal and human cases - causes fatal disease if not detected and diagnosed in time. Several divergent RABV and related lyssavirus strains have recently been reported. Given the importance of timely post-exposure prophylaxis, detecting the presence of the virus in infected animals and exposed humans is crucial. This was highlighted in a recent case from Australia ${ }^{1}$, in which RABV primers that were designed to recognize commonly circulating strains of RABV and the related Australian bat lyssavirus (ABL) did not detect a divergent ABL strain in a child who had been bitten by a bat, and a correct diagnosis was not possible until the disease had progressed beyond all hope of effective treatment. There is also growing evidence that polymorphisms in the RABV genome can affect virulence and transmission. Two recent studies highlight the need for whole-genome sequencing of circulating RABV and related lyssaviruses.

Whole-genome sequences of RABV can be used to detect cross-species transmission. Researchers in California studied samples from a 2009 rabies outbreak in foxes that had been infected with a RABV variant that usually circulates in skunks². Using non-passaged virus from archived skunk and fox tissue samples that had been collected over a 15 year period, the authors sequenced the full genomes at high depth and conducted phylogenetic and rare-variant analyses. Many of the mutations in the consensus sequences of the outbreak viruses were present at low frequencies in the historical samples. Of all low frequency mutations that were found in the historical samples, the eventual 'outbreak' mutations were present at higher frequencies than variants than were not associated with an outbreak. Eleven amino acid inversion sites were detected between historical and outbreak samples, and one mutation could be used to distinguish between samples that were taken from animals in the north of the study site and samples that were taken from animals in the south. The skunk (donor host) viral sequences could not be readily differentiated from the fox (recipient host) sequences, which indicates that there is relatively equal transmission by both foxes and skunks. The authors reported an unusual sequence in the final four amino acids at the carboxyl end of the $G$ protein (the glycoprotein on the virus surface, which has a major role in pathogenesis), although they reported no obvious impact on virulence.

Researchers from the Wuhan Institute of Virology ${ }^{3}$ used a different approach to amplify whole RABV genomes, which also
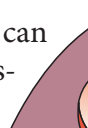

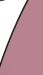

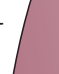

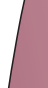

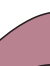

revealed important coding changes 7 that may influence virulence and 3 transmission. Beginning with strains from dogs in China and a human rabies case from Mexico, they passaged the viruses through neonatal mouse brains. They amplified whole genomes from the isolates using 5' RACE (rapid amplification of cDNA ends) and a combination of ten overlapping RT-PCR primer pairs that were designed to match a RABV consensus sequence based on two known divergent strains. The amplicons were then sequenced using Sanger sequencing.
When the amino acid sequences of the G proteins were compared, they found several changes. These are of particular interest, as changes in the $G$ protein have been reported to affect the invasiveness and pathogenicity of RABV. Strains of RABV that are currently circulating in dogs in China have been noted to have a shorter incubation period than other RABV strains and are replacing other circulating RABV strains. Whole-genome sequencing has the potential to identify the genetic changes that cause this new phenotype. However, it has previously been noted that passage of RABV through mice increases the RABV mutation rate in protein-coding regions ${ }^{4}$, emphasizing the value of the cultureindependent method that was developed by Borucki et al. ${ }^{2}$.

Whole-genome sequencing of RABV is important for making clinical diagnoses, tracking virus phylogenetics and monitoring changes in virulence. Given the range of RABV animal hosts and the potential for substantial virus evolution in animal reservoirs before jumping to humans, it is important to sequence whole genomes in animal reservoirs in order to monitor minor variants and mutations in genes that are associated with virulence.

Eva Archer and Charlotte J. Houldcroft are at the Sanger Institute, Wellcome Trust Genome Campus, Hinxton, Cambridge CB 10 1SA, UK. e-mail:microbes@sanger.ac.uk doi: $10.1038 /$ nrmicro3263

1. Francis, J. R. et al. Australian bat lyssavirus in a child: the first reported case. Pediatrics http://dx.doi. org/10.1542/peds.2013-1782 (2014).

2. Borucki, M. K. et al. Ultra-deep sequencing of intra-host rabies virus populations during cross-species transmission. PLoS Negl. Trop. Dis. 7, e2555 (2013).

3. Yu, F. et al. Comparison of complete genome sequences of dog rabies viruses isolated from China and Mexico reveals key amino acid changes that may be associated with virus replication and virulence. Arch. Virol. http:// dx.doi.org/10.1007/s00705-013-1966-2 (2014).

4. Kissi, B. et al. Dynamics of rabies virus quasispecies during serial passages in heterologous hosts. J. Gen. Virol. 80, 2041-2050 (1999).

Competing interests statement

The authors declare no competing interests. 